Document downloaded from:

http://hdl.handle.net/10251/44436

This paper must be cited as:

Adrián Martínez, S.; Ardid Ramírez, M.; Bou Cabo, M.; Espinosa Roselló, V.; Ferri García, M.; Larosa, G.; Llorens Alvarez, CD.... (2013). Detection potential of the KM3NeT detector for high-energy neutrinos from the Fermi bubbles. Astroparticle Physics. 42(1):7-14. doi:10.1016/j.astropartphys.2012.11.010.

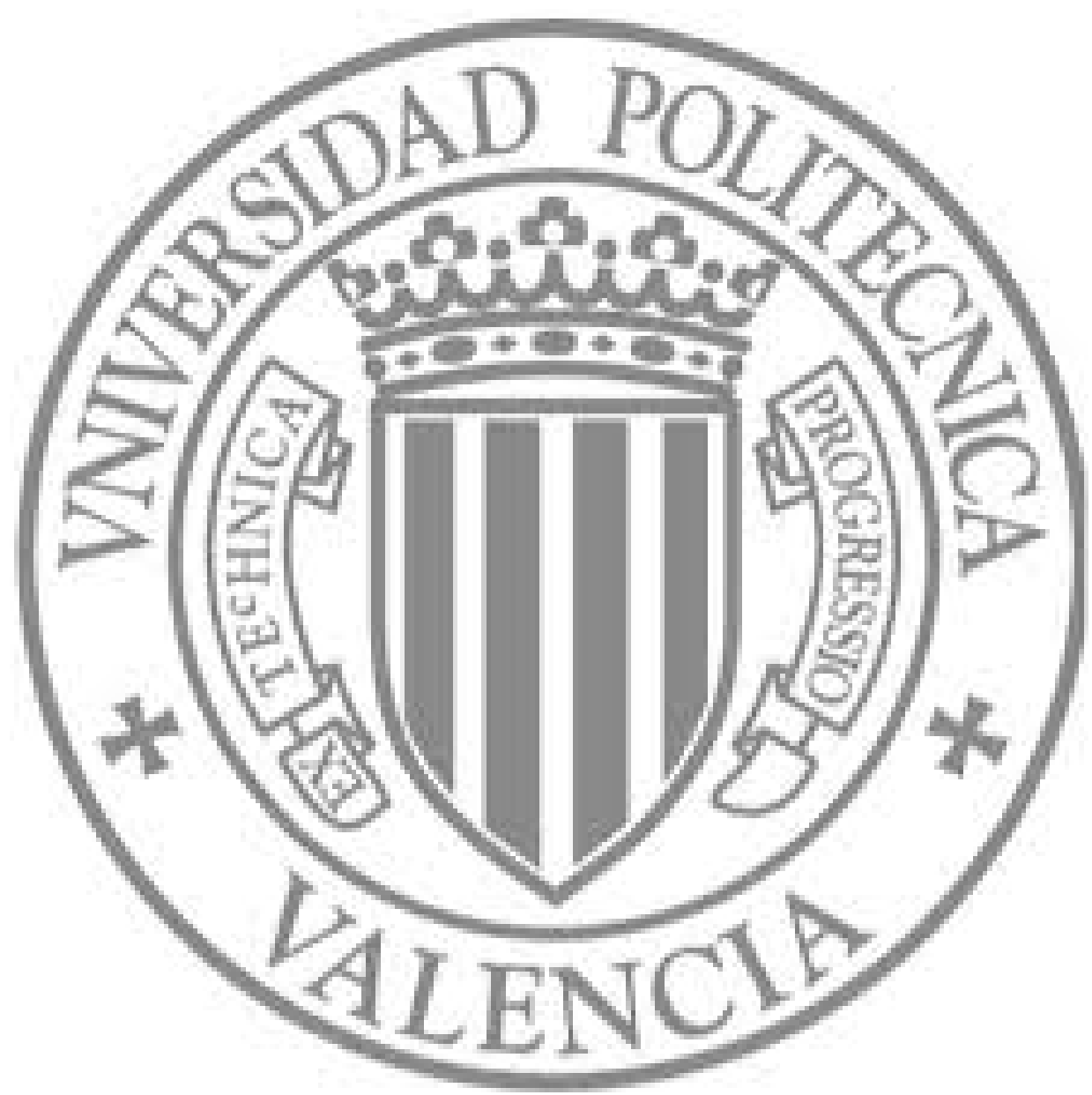

The final publication is available at

http://dx.doi.org/10.1016/j.astropartphys.2012.11.010

Copyright Elsevier 


\section{Detection Potential of the KM3NeT Detector for High-Energy Neutrinos from the Fermi Bubbles}

\section{The KM3NeT Collaboration}

S. Adrián-Martínez ${ }^{45}$, M. Ageron ${ }^{9}$, J.A. Aguilar ${ }^{16}$, F. Aharonian ${ }^{11}$, S. Aiello ${ }^{21}$, A. Albert ${ }^{13}$, M. Alexandri ${ }^{14}$, F. Ameli ${ }^{29}$, E.G. Anassontzis ${ }^{4}$, M. Anghinolfi ${ }^{23}$, G. Anton ${ }^{12}$, S. Anvar ${ }^{6}$, M. Ardid ${ }^{45}$, A. Assis Jesus ${ }^{39}$, J.-J. Aubert ${ }^{9}$, R. Bakker ${ }^{40}$, A.E. Ball ${ }^{41}$, G. Barbarino ${ }^{27}$, E. Barbarito ${ }^{18}$, F. Barbato ${ }^{27}$, B. Baret ${ }^{3}$, M. de Bel ${ }^{2}$, A. Belias ${ }^{41,14}$, N. Bellou ${ }^{34}$, E. Berbee ${ }^{39}$,

A. Berkien ${ }^{39}$, A. Bersani ${ }^{23}$, V. Bertin ${ }^{9}$, S. Beurthey $^{9}$, S. Biagi ${ }^{19}$, C. Bigongiari ${ }^{16}$, B. Bigourdan ${ }^{17}$, M. Billault ${ }^{9}$, R. de Boer ${ }^{39}$, H. Boer Rookhuizen ${ }^{39}$, M. Bonori ${ }^{30}$, M. Borghini ${ }^{8}$, M. Bou-Cabo ${ }^{45}$, B. Bouhadef ${ }^{28}$, G. Bourlis ${ }^{15}$, M. Bouwhuis ${ }^{39}$, S. Bradbury ${ }^{36}$, A. Brown ${ }^{9}$, F. Bruni ${ }^{43}$, J. Brunner ${ }^{9}$, M. Brunoldi ${ }^{23}$, J. Busto ${ }^{9}$, G. Cacopardo ${ }^{24}$,

L. Caillat ${ }^{9}$, D. Calvo Díaz-Aldagalán ${ }^{16}$, A. Calzas ${ }^{9}$, M. Canals ${ }^{5}$, A. Capone ${ }^{30}$, J. Carr ${ }^{9}$, E. Castorina ${ }^{28}$, S. Cecchini $^{19}$, A. Ceres ${ }^{18}$, R. Cereseto ${ }^{23}$, Th. Chaleil ${ }^{6}$, F. Chateau ${ }^{6}$, T. Chiarusi ${ }^{19}$, D. Choqueuse ${ }^{17}$, P.E. Christopoulou ${ }^{15}$, G. Chronis $^{14}$, O. Ciaffoni ${ }^{25}$, M. Circella ${ }^{18}$, R. Cocimano $^{24}$, F. Cohen ${ }^{13}$, F. Colijn ${ }^{34}$, R. Coniglione ${ }^{24, \star}$, M. Cordelli $^{25}$,

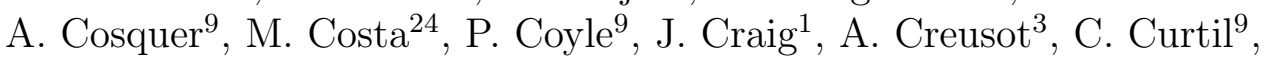
A. D'Amico ${ }^{24}$, G. Damy ${ }^{17}$, R. De Asmundis ${ }^{26}$, G. De Bonis ${ }^{30}$, G. Decock ${ }^{6}$, P. Decowski ${ }^{39}$, E. Delagnes ${ }^{6}$, G. De Rosa ${ }^{27}$, C. Distefano ${ }^{24}$, C. Donzaud ${ }^{3, a}$, D. Dornic ${ }^{9}$, Q. Dorosti-Hasankiadeh ${ }^{35}$, J. Drogou ${ }^{17}$, D. Drouhin ${ }^{13}$, F. Druillole ${ }^{6}$, L. Drury $^{11}$, D. Durand ${ }^{6}$, G.A. Durand ${ }^{6}$, T. Eberl ${ }^{12}$, U. Emanuele ${ }^{16}$, A. Enzenhöfer ${ }^{12}$, J.-P. Ernenwein ${ }^{9}$, S. Escoffier ${ }^{9}$,

V. Espinosa ${ }^{45}$, G. Etiope ${ }^{31}$, P. Favali ${ }^{31}$, D. Felea ${ }^{33}$, M. Ferri ${ }^{45}$, S. Ferry ${ }^{6}$,

V. Flaminio ${ }^{28}$, F. Folger ${ }^{12}$, A. Fotiou ${ }^{41}$, U. Fritsch ${ }^{12}$, D. Gajanana ${ }^{39}$, R. Garaguso ${ }^{28}$, G.P. Gasparini ${ }^{8}$, F. Gasparoni ${ }^{43}$, V. Gautard ${ }^{6}$,

F. Gensolen ${ }^{9}$, K. Geyer ${ }^{12}$, G. Giacomelli ${ }^{19}$, I. Gialas ${ }^{15}$, V. Giordano ${ }^{24}$, J. Giraud ${ }^{6}$, N. Gizani ${ }^{15}$, A. Gleixner ${ }^{12}$, C. Gojak ${ }^{9}$, J.P. Gómez-González ${ }^{16}$, K. Graf ${ }^{12}$, D. Grasso ${ }^{28}$, A. Grimaldi ${ }^{21}$, R. Groenewegen ${ }^{40}$, Z. Guédé ${ }^{17}$, G. Guillard ${ }^{32}$, F. Guilloux ${ }^{6}$, R. Habel ${ }^{25}$, G. Hallewell ${ }^{9}$, H. van Haren ${ }^{40}$, J. van Heerwaarden ${ }^{40}$, A. Heijboer ${ }^{39}$, E. Heine ${ }^{39}$, J.J. Hernández-Rey ${ }^{16}$, B. Herold ${ }^{12}$, T. Hillebrand ${ }^{40}$, M. van de Hoek ${ }^{39}$, J. Hogenbirk ${ }^{39}$, J. Höß1², C.C. $\mathrm{Hsu}^{39}$, M. Imbesi ${ }^{24}$, A. Jamieson ${ }^{1}$, P. Jansweijer ${ }^{39}$, M. de Jong ${ }^{39}$, F. Jouvenot ${ }^{37}$, M. Kadler ${ }^{12, b}$, N. Kalantar-Nayestanaki ${ }^{35}$, O. Kalekin ${ }^{12}$, 
A. Kappes ${ }^{12, c}$, M. Karolak ${ }^{6}$, U.F. Katz ${ }^{12}$, O. Kavatsyuk ${ }^{35}$, P. Keller ${ }^{9}$, Y. Kiskiras ${ }^{41}$, R. Klein ${ }^{12}$, H. Kok ${ }^{39}$, H. Kontoyiannis ${ }^{14}$, P. Kooijman ${ }^{2,39,44}$, J. Koopstra ${ }^{2,39}$, C. Kopper ${ }^{39, d}$, A. Korporaal ${ }^{39}$, P. Koske ${ }^{34}$, A. Kouchner ${ }^{3}$,

S. Koutsoukos ${ }^{4}$, I. Kreykenbohm ${ }^{12}$, V. Kulikovskiy ${ }^{23, e}$, M. Laan ${ }^{40}$, C. La Fratta ${ }^{31}$, P. Lagier ${ }^{9}$, R. Lahmann ${ }^{12}$, P. Lamare ${ }^{9}$, G. Larosa ${ }^{45}$, D. Lattuada ${ }^{24}$, A. Leisos ${ }^{15}$, D. Lenis ${ }^{15}$, E. Leonora ${ }^{21}$, H. Le Provost ${ }^{6}$, G. Lim $^{2}$, C.D. Llorens ${ }^{45}$, J. Lloret ${ }^{45}$, H. Löhner ${ }^{35}$, D. Lo Presti ${ }^{22}$, P. Lotrus ${ }^{6}$,

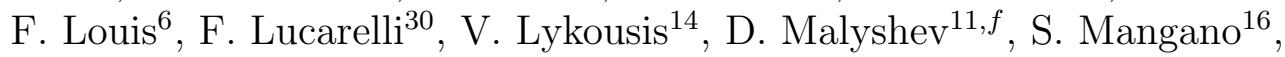
E.C. Marcoulaki ${ }^{38}$, A. Margiotta ${ }^{19}$, G. Marinaro ${ }^{31}$, A. Marinelli ${ }^{28}$, O. Maris ${ }^{33}$, E. Markopoulos ${ }^{41}$, C. Markou ${ }^{38}$, J.A. Martínez-Mora ${ }^{45}$, A. Martini ${ }^{25}$, J. Marvaldi ${ }^{17}$, R. Masullo ${ }^{30}$, G. Maurin ${ }^{6, g}$, P. Migliozzi $^{26}$, E. Migneco ${ }^{24}$, S. Minutolii ${ }^{23}$, A. Miraglia ${ }^{24}$, C.M. Mollo ${ }^{26}$, M. Mongelli ${ }^{18}$, E. Monmarthe ${ }^{6}$, M. Morgantiti ${ }^{28}, \mathrm{~S} . \mathrm{Mos}^{39}, \mathrm{H} . \mathrm{Motz}^{12, h}$, Y. Moudden ${ }^{6}$,

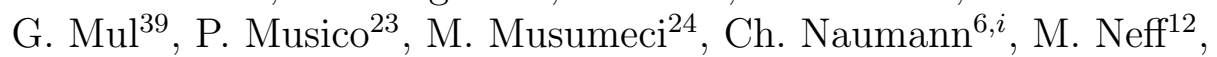
C. Nicolaou $^{10}$, A. Orlando ${ }^{24}$, D. Palioselitis ${ }^{39}$, K. Papageorgiou ${ }^{15}$,

A. Papaikonomou ${ }^{4}$, R. Papaleo ${ }^{24}$, I.A. Papazoglou ${ }^{38}$, G.E. Păvălaş ${ }^{33}$, H.Z. Peek ${ }^{39}$, J. Perkin ${ }^{42}$, P. Piattellii ${ }^{24}$, V. Popa ${ }^{33}$, T. Pradier ${ }^{32}$,

E. Presani ${ }^{39}$, I.G. Priede ${ }^{1}$, A. Psallidas ${ }^{41}$, C. Rabouille ${ }^{7}$, C. Racca ${ }^{13}$, A. $\operatorname{Radu}^{33}$, N. Randazzo ${ }^{21}$, P.A. Rapidis ${ }^{38}$, P. Razis ${ }^{10}$, D. Real ${ }^{16}$, C. Reed $^{39}$, S. Reito ${ }^{21}$, L.K. Resvanis ${ }^{4,41}$, G. Riccobene ${ }^{24}$, R. Richter ${ }^{12}$, K. Roensch ${ }^{12}$, J. Rolin ${ }^{17}$, J. Rose ${ }^{36}$, J. Roux ${ }^{9}$, A. Rovelli ${ }^{24}$, A. Russo ${ }^{27}$, G.V. Russo ${ }^{22}$, F. Salesa ${ }^{16}$, D. Samtleben ${ }^{39}$, P. Sapienza ${ }^{24}$, J.-W. Schmelling ${ }^{39}$, J. Schmid ${ }^{12}$, J. Schnabel ${ }^{12}$, K. Schroeder ${ }^{8}$, J.-P. Schuller ${ }^{6}$, F. Schussler ${ }^{6}$, D. Sciliberto ${ }^{21}$,

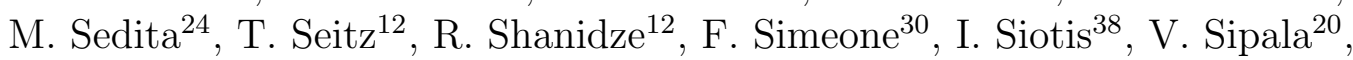

C. Sollima ${ }^{28}$, S. Sparnocchia ${ }^{8}$, A. Spies ${ }^{12}$, M. Spurio ${ }^{19}$, T. Staller ${ }^{34}$, S. Stavrakakis ${ }^{14}$, G. Stavropoulos ${ }^{41}$, J. Steijger ${ }^{39}$, Th. Stolarczyk ${ }^{6}$, D. Stransky ${ }^{12}$, M. Taiuti ${ }^{23}$, A. Taylor ${ }^{11}$, L. Thompson ${ }^{42}$, P. Timmer ${ }^{39}$, D. Tonoiu ${ }^{33}$, S. Toscano ${ }^{16}$, C. Touramanis ${ }^{37}$, L. Trasatti $^{25}$, P. Traverso ${ }^{8}$, A. Trovato $^{24}$, A. Tsirigotis ${ }^{15}$, S. Tzamarias ${ }^{15}$, E. Tzamariudaki ${ }^{38}$, F. Urbano ${ }^{16}$, B. Vallage ${ }^{6}$, V. Van Elewyck ${ }^{3}$, G. Vannoni ${ }^{6}$, M. Vecchi ${ }^{9}$, P. Vernin ${ }^{6}$, S. Viola ${ }^{24}$, D. Vivolo ${ }^{27}$, S. Wagner ${ }^{12}$, P. Werneke ${ }^{39}$, R.J. White ${ }^{36}$, G. Wijnker ${ }^{39}$, J. Wilms ${ }^{12}$, E. de Wolf ${ }^{2,39}$, H. Yepes ${ }^{16}$, V. Zhukov ${ }^{41}$, E. Zonca ${ }^{6}$, J.D. Zornoza ${ }^{16}$, J. Zúñiga ${ }^{16}$

\footnotetext{
University of Aberdeen, United Kingdom

University of Amsterdam, the Netherlands

3 APC - AstroParticule et Cosmologie - UMR 7164 (CNRS, Université

Paris 7, CEA, Observatoire de Paris), Paris, France
} 
University of Athens, Greece

University of Barcelona, Spain

CEA, IRFU, Centre de Saclay, 91191 Gif-sur-Yvette, France

CEA-CNRS-UVSQ, LSCE/IPSL, 91198 Gif-sur-Yvette, France

CNR-ISMAR, La Spezia, Trieste, Genova, Italy

CPPM, Aix-Marseille Université, CNRS/IN2P3, Marseille, France

University of Cyprus

11 Dublin Institute for Advanced Studies (DIAS), Ireland

12 Erlangen Centre for Astroparticle Physics (ECAP), University of Erlangen-Nuremberg, Germany

Groupe de Recherche en Physique des Hautes Energies

(GRPHE)/EA3438/Université de Haute Alsace, Colmar, France

14 Hellenic Centre for Marine Research (HCMR), Greece

15 Hellenic Open University, Patras, Greece

16 IFIC - Instituto de Física Corpuscular, CSIC and Universitat de València, Spain

17 IFREMER, France

18 INFN Sezione di Bari and University of Bari, Italy

19 INFN Sezione di Bologna and University of Bologna, Italy

20 INFN Sezione di Cagliari and University of Sassari, Italy

21 INFN Sezione di Catania, Italy

22 INFN Sezione di Catania and University of Catania, Italy

23 INFN Sezione di Genova and University of Genova, Italy

24 INFN Laboratori Nazionali del Sud, Catania, Italy

25 INFN Laboratori Nazionali di Frascati, Italy

26 INFN Sezione di Napoli, Italy

27

INFN Sezione di Napoli and University of Napoli, Italy

INFN Sezione di Pisa and University of Pisa, Italy

INFN Sezione di Roma, Italy

INFN Sezione di Roma and University of Roma 1 "La Sapienza", Italy

Istituto Nazionale di Geofisica e Vulcanologia (INGV), Italy

University of Strasbourg and Institut Pluridisciplinaire

Hubert Curien/IN2P3/CNRS, Strasbourg, France

33

34

35

36

37

Institute of Space Science, Măgurele-Bucharest, Romania

University of Kiel, Germany

KVI, University of Groningen, the Netherlands

University of Leeds, United Kingdom

University of Liverpool, United Kingdom

National Center of Scientific Research "Demokritos", Athens, Greece

Nikhef, Amsterdam, the Netherlands 
40 Koninklijk Nederlands Instituut voor Onderzoek der Zee (NIOZ), Texel, the Netherlands

41 NOA/NESTOR, Pylos, Greece

42 University of Sheffield, United Kingdom

43 Tecnomare, Ven, Italy

44 University of Utrecht, the Netherlands

45 Institut d'Investigació per a la Gestió integrada de les Zones Costaneres, Universitat Politècnica València, Gandia, Spain

$a \quad$ also at Université Paris-Sud, 91405 Orsay Cedex, France

$b$ now at Universität Würzburg, Germany

$c$ on leave of absence at Humboldt University, Berlin, Germany

$d$ now at Dept. of Physics and Wisconsin IceCube Particle Astrophysics Center, University of Wisconsin, Madison, WI 53706, USA

$e$ also at Moscow State University, Skobeltsyn Institute of Nuclear Physics, Moscow, Russia

$f$ now at Bogolyubov Institute for Theoretical Physics, Ukraine

$g$ now at Laboratoire d'Annecy-le-Vieux de physique des particules (LAPP), France

$h$ now at Institut for Cosmic Ray Research, University of Tokyo, Japan

$i$ now at Université Paris VI, Laboratoire de Physique Nucléaire et de Hautes Energies (LPNHE), France

* corresponding author, e-mail address: coniglione@lns.infn.it

\section{Abstract}

A recent analysis of the Fermi Large Area Telescope data provided evidence for a high-intensity emission of high-energy gamma rays with a $E^{-2}$ spectrum from two large areas, spanning $50^{\circ}$ above and below the Galactic centre (the "Fermi bubbles"). A hadronic mechanism was proposed for this gamma-ray emission making the Fermi bubbles promising source candidates of high-energy neutrino emission. In this work Monte Carlo simulations regarding the detectability of high-energy neutrinos from the Fermi bubbles 
with the future multi- $\mathrm{km}^{3}$ neutrino telescope KM3NeT in the Mediterranean Sea are presented. Under the hypothesis that the gamma-ray emission is completely due to hadronic processes, the results indicate that neutrinos from the bubbles could be discovered in about one year of operation, for a neutrino spectrum with a cutoff at $100 \mathrm{TeV}$ and a detector with about $6 \mathrm{~km}^{3}$ of instrumented volume. The effect of a possible lower cutoff is also considered. Keywords: neutrino telescope, Fermi Bubbles, KM3NeT

\section{Introduction}

In the last decade a new generation of telescopes revealed a large variety of astrophysical high-energy gamma-ray sources. Telescopes such as Fermi [1], HESS [2], VERITAS [3] and MAGIC [4] can identify sources of gamma rays with energies from about $20 \mathrm{MeV}$ to about $100 \mathrm{TeV}$ with sufficient angular resolution to study the source morphology of extended Galactic sources. They can also measure the gamma-ray energy spectrum with high precision. From these measurements the presence of cosmic acceleration processes has been confirmed in a large variety of known and unknown galactic and extragalactic sources [5, 6].

For the full understanding of the underlying mechanisms other probes that can complement the information from gamma-ray detection are needed. As neutrinos are mainly produced via proton-proton $(p p)$ and proton-gamma $(p \gamma)$ interactions, they are an unambiguous signature of hadronic acceleration.

Neutrino telescopes have been constructed, and more sensitive ones are proposed, to detect these high-energy neutrinos. Neutrino flux estimates 
indicate that detectors of $\mathrm{km}^{3}$-scale instrumented volume are required [7]. The IceCube [8] detector located at the South Pole, about $1 \mathrm{~km}^{3}$ in size, is taking data in its final configuration since 2011. In the northern hemisphere ANTARES is the largest operating detector located in the Mediterranean Sea $40 \mathrm{~km}$ off the French coast near Toulon [9]. It has an instrumented volume of about $0.01 \mathrm{~km}^{3}$ and it is taking data in its final configuration since 2008 . The KM3NeT consortium [10] proposes the construction of a multi-km ${ }^{3}$ telescope in the Mediterranean Sea (KM3NeT). From their respective locations, KM3NeT and ANTARES will detect upward-going neutrinos from about $3.5 \pi$ sr of the sky, including the Galactic centre and most of the Galactic plane, where many TeV gamma-ray sources are located [5, 7].

In this work the response of the KM3NeT telescope to neutrinos from the Fermi bubbles (see below) is studied with Monte Carlo simulations under the hypothesis that a hadronic mechanism is responsible for the gammaray emission. In particular, the neutrino flux that could be discovered by KM3NeT is determined as a function of the number of observation years.

\section{The Fermi bubbles}

A recent analysis of Fermi-LAT data [11] revealed an intense gamma-ray emission from two large areas above and below the Galactic centre. The detected gamma-ray emission has the following characteristics:

- The emission areas are symmetric with respect to the Galactic plane and extend up to 50 degrees $(10 \mathrm{kpc})$ north and south the Galactic centre, with a width of 40 degrees in Galactic longitude. 
- The gamma energy spectrum, measured from $\sim 1 \mathrm{GeV}$ to $\sim 100 \mathrm{GeV}$, is compatible with a power-law spectrum described by $E^{2} d \Phi_{\gamma} / d E \approx$ $3-6 \times 10^{-7} \mathrm{GeV} \mathrm{cm}^{-2} \mathrm{~s}^{-1} \mathrm{sr}^{-1}$.

- The emission is homogeneous within the bubbles. No significant differences were found between the northern and the southern bubble.

- The edges of the bubbles seem to be correlated with ROSAT X-ray maps at $1.5-2 \mathrm{keV}$, while the inner parts are correlated with the hardspectrum microwave excess known as WMAP haze [11, 12, 13].

Several mechanisms have been suggested in the literature to explain this gamma-ray emission. Most of these explanations rely on leptonic processes and include inverse Compton scattering by electrons, either produced by long-lasting energy injections near the Galactic centre [11] or accelerated through the second-order Fermi process generated by turbulent plasma throughout the entire bubble [14]. Other processes such as recent transient AGN activity near the Galactic centre [15], millisecond pulsars [16] and dark matter annihilation [17] have also been put forward.

Currently the observed features cannot be fully explained by these predominantly leptonic processes, which has led to a proposal for an underlying hadronic process [18]. A cosmic ray population associated with long-timescale star formation in the Galactic centre (of the order of $10^{10}$ years) was hypothesised to have been injected into the bubbles where it interacts with the ambient matter and produces high-energy gamma rays through $\pi^{0}$ decay. Pair production is responsible for the generation of secondary electrons producing the synchrotron radiation observed as microwaves. In this scenario 
neutrinos are produced through the decay of charged pions. The leptonic and hadronic mechanisms require very different galactic diffusion characteristics.

Recently the morphological and spectral characteristics of the WMAP haze have been reviewed [13], with important implications for the possible production scenarios. The author considers all the proposed models to explain the gamma and the WMAP haze emission and concludes that hybrid emission scenarios will likely be required.

The detection of high-energy neutrinos can distinguish between hadronic and leptonic models. Only if a hadronic process is totally or partially responsible for the production of gamma rays from the Fermi bubbles, neutrinos will also be produced. The KM3NeT telescope, to be located in the deep Mediterranean Sea, will be the ideal instrument for the observation of neutrinos from the Fermi bubbles.

\section{The detector}

The KM3NeT consortium aims at installing a deep-sea research infrastructure hosting a multi-cubic-kilometre high-energy neutrino detector in the Mediterranean Sea. During the Design Study 1 , several technical solutions were investigated and the results reported in the Technical Design Report [20]. During a Preparatory Phase ${ }^{2}$, a final design concept was defined. Currently prototyping activities are in progress to prepare for the construction phase.

The detection principle relies on the detection of Cherenkov light induced

\footnotetext{
${ }^{1}$ Supported by the EU in FP6, contract $n^{\circ} 011937$

${ }^{2}$ Supported by the EU in FP7, grant agreement $n^{\circ} 212525$
} 
by secondary charged particles produced in neutrino-nucleus interactions inside or near the detector. The Cherenkov light is detected by photomultiplier tubes (PMTs) contained in glass spheres that are designed to resist the hydrostatic pressure of the deep-sea environment. These instrumented spheres are called Optical Modules (OMs).

The geometry of the detector [21] simulated in this work consists of a three-dimensional array of OMs attached to vertical structures (Detection Units, DUs). An array of DUs constitutes a detector building block. Several building blocks form the full detector of about 300 DUs. A DU, which is anchored to the sea floor and kept upright by a submerged buoy, consists of horizontal bars equipped with 2 OMs, one at each end. Adjacent bars are oriented orthogonally to each other. The DUs are connected to shore by an electro-optical cable. Each OM consists of 31 3-inch PMTs [22] housed inside a 17-inch pressure-resistant glass sphere covering almost a $4 \pi$ field of view.

For this work, a detector of 308 DUs (two building blocks of 154 DUs each, arranged uniformly in a circular area) with an average separation between adjacent DUs of $180 \mathrm{~m}$ has been simulated. Each simulated DU consists of 20 bars of $6 \mathrm{~m}$ length with a vertical spacing of $40 \mathrm{~m}$ (40 OMs per DU). The total instrumented detector volume is about $6 \mathrm{~km}^{3}$.

\section{Monte Carlo simulation}

In order to study the sensitivity of the KM3NeT telescope for the detection of neutrinos from the Fermi bubbles, we use a Monte Carlo simulation framework. The framework is based on the ANTARES software [23],

modified for a $\mathrm{km}^{3}$-scale detector and using OM properties appropriate for 
KM3NeT. The simulation chain consists of the generation of muon neutrinos from the bubbles, the generation of atmospheric muon and neutrino backgrounds, the neutrino charged-current interactions, the propagation of the produced muons in rock and sea water, the generation of Cherenkov light, the ${ }^{40} \mathrm{~K}$ background and the digitisation of the PMT signals. Optical properties of the sea water and the PMT characteristics are taken into account in the simulation. The depth and the optical water properties measured at the Sicilian Capo Passero site have been used [20]. Background light due to the presence of ${ }^{40} \mathrm{~K}$ in salt water has been simulated adding an uncorrelated hit rate of $5 \mathrm{kHz}$ per $\mathrm{PMT}$ and a time-correlated hit rate of $500 \mathrm{~Hz}$ per OM (two coincident hits in different PMTs inside the same OM). These parameters have been estimated with a complete simulation based on GEANT4 [24]. The $5 \mathrm{kHz}$ of uncorrelated hit rate is consistent with the baseline of $50 \mathrm{kHz}$ measured with the 10-inch PMTs of the ANTARES experiment [25].

The muon track direction is reconstructed from the simulated arrival times of Cherenkov photons and the PMT positions. The reconstruction algorithm is based on a hit selection and on a maximum likelihood fit that uses probability density functions for the photon arrival times at the PMTs. In addition to the positions and track directions, the number of hits used for the reconstruction $\left(N_{\text {hit }}\right)$ and a track fit quality parameter $(\Lambda)$ are given as output. The $\Lambda$ parameter is determined from the likelihood and from the number of compatible track solutions found by the algorithm and is used to reject badly reconstructed events [26]. The $N_{\text {hit }}$ parameter is correlated with the muon energy. 


\subsection{Neutrinos from the Fermi bubbles}

Muon neutrinos from the Fermi bubbles were generated homogeneously within two circular regions of $19^{\circ}$ radius around two positions in the sky at the equatorial coordinates declination $\delta=-15^{\circ}$ and right ascension $\alpha=243^{\circ}$ for the northern bubble and $\delta=-44^{\circ}$ and $\alpha=298^{\circ}$ for the southern bubble. The simulated neutrino energy is between $10^{2}$ and $10^{8} \mathrm{GeV}$.

Figure 1 shows the percentage of Monte Carlo neutrinos that are up-going in local detector coordinates. Since only up-going events can be unambiguously classified as neutrino candidates, Fig. 1 represents the Fermi bubbles' visibility for a neutrino telescope in the Mediterranean Sea. Black points are the edges of the two bubbles as reported in [11. The average visibility for a detector located at a latitude of $36^{\circ} 16^{\prime} \mathrm{N}$ is $58 \%$ of the time for the northern bubble and $80 \%$ for the southern bubble. We note that for IceCube only a small fraction of the Fermi bubbles lies below the horizon (solid red line in Fig. 1), leading to a significantly reduced sensitivity compared to a similar detector in the Mediterranean Sea [27].

The generated Monte Carlo events can be weighted to reproduce different assumed neutrino spectra. The general energy dependence of the neutrino flux used in this analysis is a power law spectrum with a spectral index of -2 and an exponential cutoff:

$$
\frac{d \Phi_{\nu}^{E_{c}}}{d E}=K_{0 \nu} \cdot E^{-2} \cdot e^{-E / E_{c}}
$$

where $E_{c}$ is the cutoff energy. For this analysis we consider three cases: $d \Phi_{\nu}^{\infty} / d E$, i.e. a pure power law spectrum with spectral index -2 , as well as $d \Phi_{\nu}^{100} / d E$ and $d \Phi_{\nu}^{30} / d E$ with cutoffs at $100 \mathrm{TeV}$ and $30 \mathrm{TeV}$, respectively. 
The cutoff at $E_{c}=100 \mathrm{TeV}$ is consistent with an assumed cutoff of the proton energy distribution in the Fermi bubbles at a few PeV (corresponding to the position of the "knee" in the cosmic ray energy spectrum). The second cutoff value considered, $E_{c}=30 \mathrm{TeV}$, is more conservative and does not require the assumption that the Fermi bubbles populate the cosmic ray spectrum up to the knee.

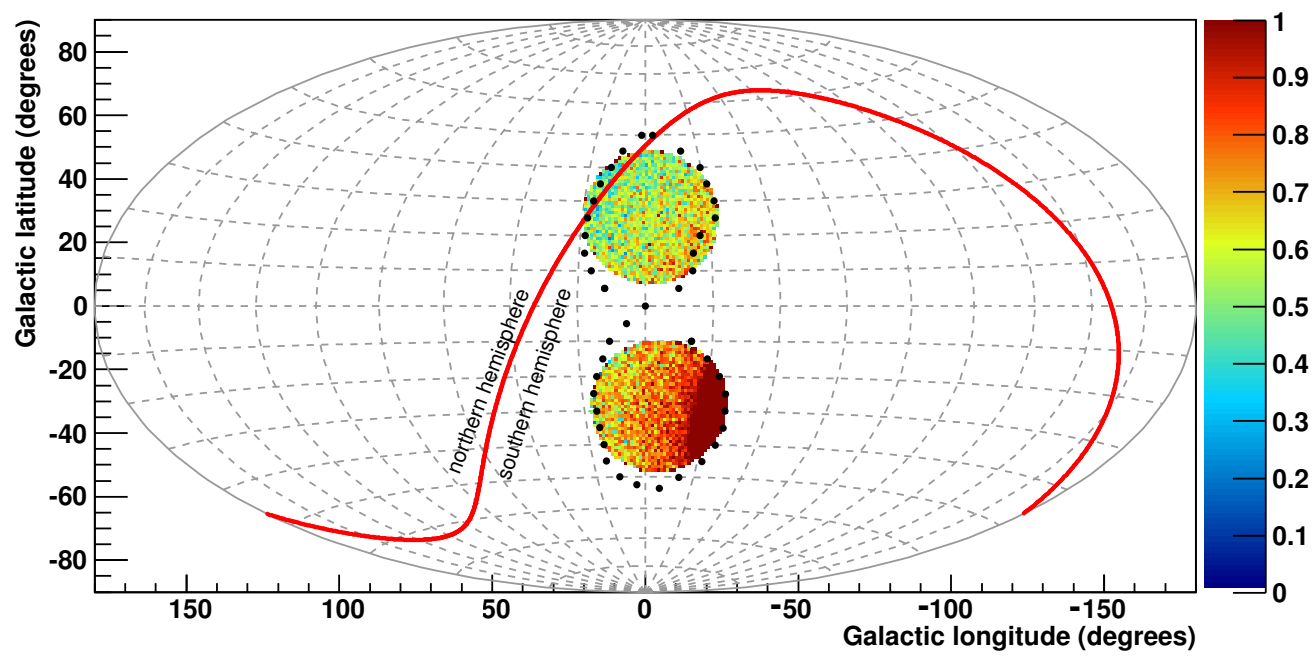

Figure 1: Galactic coordinates of simulated neutrino from the Fermi bubbles. The color code indicates the fraction of Monte Carlo neutrinos that are up-going in local detector coordinates. The measured bubble edges (11] Table 1) are also reported (black points). The red line represents the separation line between the northern and southern hemisphere.

Under the hypothesis that the source is transparent to gamma rays and that the mechanism responsible for the gamma-ray emission is hadronic, the neutrino spectrum was estimated from the measured gamma-ray spectrum following the prescription described in [19]. The measured Fermi bubble gamma-ray spectrum is consistent with a $E^{-2}$ shape and a normalisation 
$K_{0 \gamma} \approx 4 \times 10^{-7} \mathrm{GeV} \mathrm{cm}^{-2} \mathrm{~s}^{-1} \mathrm{sr}^{-1}$. Using this gamma-ray flux and taking into account the $0.7 \mathrm{sr}$ solid angle of the two simulated bubbles, we estimated the corresponding muon neutrino plus antineutrino flux to have $K_{0 \nu} \approx 1 \times$ $10^{-7} \mathrm{GeV} \mathrm{cm}^{-2} \mathrm{~s}^{-1}$.

\subsection{Atmospheric muons}

Cosmic rays entering the atmosphere produce extensive air showers that contain high-energy muons. Although the sea water above the detector serves as a shield, many of these muons reach the detector. Therefore down-going tracks are excluded from the analysis. However, atmospheric muons misreconstructed as up-going remain a significant background. Simulating atmospheric muon background in a large detector requires huge amounts of CPU time and data storage. Atmospheric muons were generated with the fast MUPAGE code [28]. This code, which is based on a full Monte Carlo simulation of primary cosmic ray interactions and shower propagation in the atmosphere, provides a parameterisation of the underwater flux of atmospheric muons including also multi-muon events ("muon bundles").

Atmospheric muons were generated in the range $1 \mathrm{TeV} \leq E_{b}<10 \mathrm{TeV}$, where $E_{b}$ is the sum of the energies of all single muons in the bundle. This sample is statistically equivalent to a live time of 2 hours. To efficiently increase statistics in the high-energy region an additional sample equivalent to 8 days of live time was generated with $E_{b} \geq 10 \mathrm{TeV}$. The Monte Carlo samples were then reweighted to the relevant experimental live time. 


\subsection{Atmospheric neutrinos}

A large number of secondary particles is produced in a cosmic ray interaction in the atmosphere. The production of pions and kaons and their subsequent decay chains produce a large flux of atmospheric neutrinos, called "conventional flux", with an energy distribution proportional to $E_{\nu}^{-3.7}$ at neutrino energies $E_{\nu}$ larger than about $1 \mathrm{TeV}$. The production of neutrinos from heavy quark is responsible for a high-energy component $\left(E_{\nu} \geq 10 \mathrm{TeV}\right)$ in the atmospheric neutrino spectrum. This contribution, called "prompt", is not well known, but several predictions are available [29, 30]. The atmospheric neutrino flux is an irreducible background for the detection of neutrinos of cosmic origin.

The atmospheric muon neutrino and antineutrino background was generated in the energy range $10^{2} \mathrm{GeV} \leq E_{\nu} \leq 10^{8} \mathrm{GeV}$ and over the full solid angle. The events are weighted to reproduce the conventional atmospheric neutrino flux following the Bartol model [31]. The uncertainty on the Bartol normalisation factor is taken to be about 25-30\% [32. A prompt contribution is also taken into account through the event weights. The models in [29, 30] have been considered and the model with the highest neutrino flux, corresponding to the highest prediction of the Recombination Quark Parton Model (RQPM) [33, has been used in the present analysis.

\section{Flux discovery potential}

The present analysis was restricted to events reconstructed as up-going and located within $19^{\circ}$ around the centre of each Fermi bubble. The bubbles are considered as discovered if the number of detected events in a given 
detector live time has a probability of $\alpha=2.85 \times 10^{-7}$ or less to originate purely from background in $1-\beta=50 \%$ of all experiments. This corresponds to a significance of $5 \sigma$ (area of the one-sided Gaussian tail).

The signal flux required to claim a discovery is calculated from the simulated average number of background events, $\left\langle n_{\text {back }}\right\rangle$. First, the minimum (critical) number of events, $n_{0}$, that satisfies

$$
\sum_{n_{\mathrm{obs}}=n_{0}}^{\infty} P\left(n_{\mathrm{obs}} \mid\left\langle n_{\text {back }}\right\rangle\right)<\alpha
$$

is determined, where $P\left(n_{\text {obs }} \mid\left\langle n_{\text {back }}\right\rangle\right)$ is the Poisson probability for observing $n_{\text {obs }}$ events given $\left\langle n_{\text {back }}\right\rangle$. The value $n_{0}$ is the minimum number of events required to claim a deviation from the background-only hypothesis with a statistical significance defined by the $p$-value $\alpha$. The confidence level C.L. is related to the $p$-value via C.L. $=1-\alpha$.

In case of the presence of a signal of strength $n_{\alpha}$, the probability to observe $n_{0}$ or more events is related to the statistical power $(1-\beta)$ by

$$
\sum_{n_{\mathrm{obs}}=n_{0}}^{\infty} P\left(n_{\mathrm{obs}} \mid\left\langle n_{\alpha}\right\rangle+\left\langle n_{\text {back }}\right\rangle\right)=1-\beta .
$$

The signal strength $n_{\alpha}\left(\left\langle n_{\text {back }}\right\rangle\right)$ resulting from (3) would lead to an observation with a $p$-value less than $\alpha$ in a fraction $(1-\beta)$ of the experiments.

In order to determine the minimum flux $\phi_{\alpha}$ needed for a discovery (discovery flux), the cuts on $\Lambda$ and $N_{\text {hit }}$ were varied and the minimum in the Model Discovery Potential (MDP) 34], defined as MDP $=n_{\alpha}\left(\left\langle n_{\text {back }}\right\rangle\right) /\left\langle n_{s}\right\rangle$ was sought. Here, $\left\langle n_{s}\right\rangle$ is the number of signal events resulting from the model flux (1) with $K_{0 \nu} \approx 1 \times 10^{-7} \mathrm{GeV} \mathrm{cm}^{-2} \mathrm{~s}^{-1}$ after cuts. The discovery 
flux is then related to the MDP by

$$
\phi_{\alpha}=K_{0 \nu} \cdot \mathrm{MDP}=K_{0 \nu} \cdot \frac{n_{\alpha}\left(\left\langle n_{\text {back }}\right\rangle\right)}{\left\langle n_{s}\right\rangle} .
$$

Figure 2 shows the cumulative distributions (as functions of $\Lambda$ and $N_{\text {hit }}$ ) of the numbers of events per year, reconstructed as up-going in the bubble region, for the different Monte Carlo samples. Figure 2 demonstrates that most of the events due to atmospheric muons that are misreconstructed as up-going can be rejected by imposing an appropriate cut on $\Lambda$, while an appropriate cut on $N_{\text {hit }}$ helps in the rejection of atmospheric neutrino background events 35 .
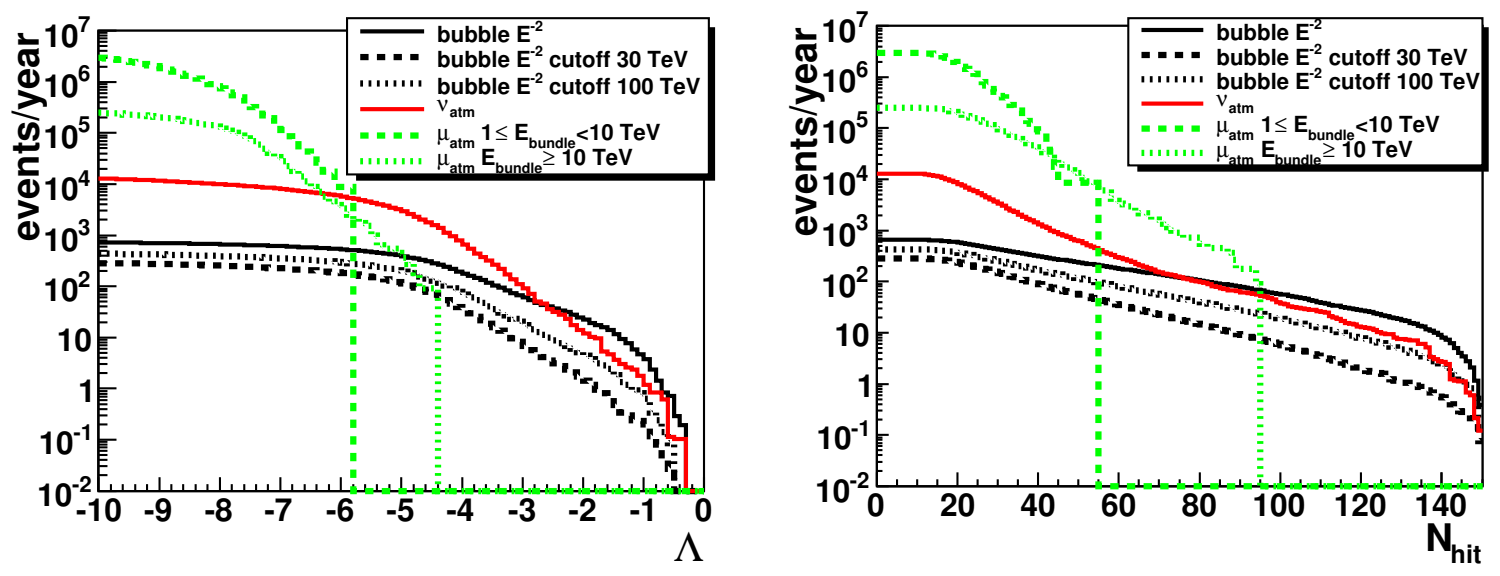

Figure 2: Cumulative distribution of the number of events per year reconstructed as up-going in the bubble region as a function of $\Lambda$ (left panel) and of $N_{\text {hit }}$ (right panel) for the different Monte Carlo samples. For the right panel a cut $\Lambda \geq-10$ was applied.

The cuts required to minimise the MDP vary for the different flux assumption and are given in Tab. 1. Figure 3 shows the spectrum of reconstructed signal and background up-going events per year in the region of the bubbles as a function of the simulated neutrino energy. The signal energy spectrum 

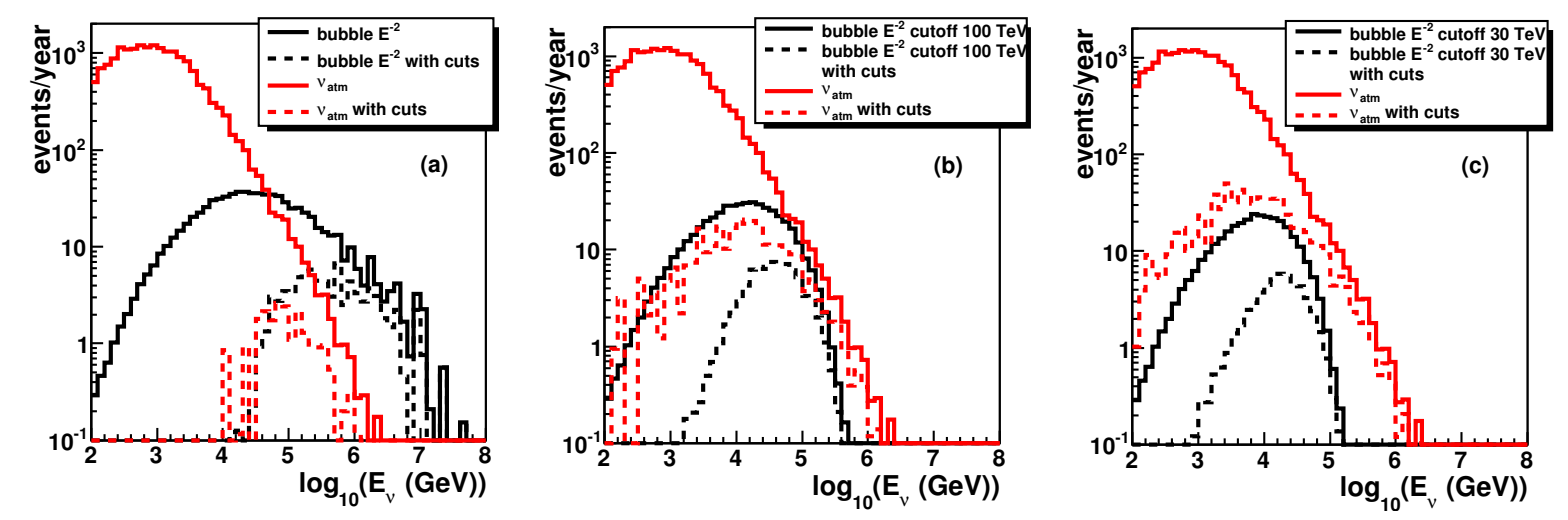

Figure 3: Number of events per year reconstructed as up-going in the bubble region as a function of the simulated neutrino energy, for the signal and the atmospheric neutrino background with and without the cuts required to minimise the MDP ( $5 \sigma$ C.L., $50 \%$ probability). The assumed normalisation factor is $K_{0 \nu}=1 \times 10^{-7} \mathrm{GeV} \mathrm{cm}^{-2} \mathrm{~s}^{-1}$. The bin width in $\log _{10}\left(E_{\nu}\right)$ is 0.1 .

from the bubbles, after the cuts, is centred at about $500 \mathrm{TeV}$ for $d \Phi_{\nu}^{\infty} / d E$ (see Eq. (1D) (a), at about $40 \mathrm{TeV}$ for $d \Phi_{\nu}^{100} / d E$ (b) and at about $25 \mathrm{TeV}$ for $d \Phi_{\nu}^{30} / d E$ (c). The numbers of events at the reconstruction level and after the cuts in one year of data taking are reported in Tab. 1 for a source with a flux normalisation factor of $K_{0 \nu}=1 \times 10^{-7} \mathrm{GeV} \mathrm{cm}^{-2} \mathrm{~s}^{-1}$.

With the small simulated live time of the atmospheric muon samples the number of events per year at cut level due to the atmospheric muons is zero (see Tab. 1). By extrapolating in these data sets the trends of the numbers of events per year as a function of $\Lambda$ and $N_{\text {hit }}$ (Fig. 2), it was estimated that, in the cases of source neutrino spectra with cutoffs, the contribution of atmospheric muons to the background does not exceed $25 \%$ of the atmospheric neutrino background events reported in Tab. 1. A 25\% increase in 


\begin{tabular}{|c|c|c|c|c|c|c|}
\hline Spectrum & & $\nu_{\text {sig }}$ & $\nu_{\text {atm }}$ & $\mu_{\text {atm }}$ & $\Lambda$ & $N_{\text {hit }}$ \\
\hline \multirow{2}{*}{$\propto E^{-2}$} & reco level & 770 & 17707 & $8 \times 10^{6}$ & & \\
\cline { 2 - 8 } & cut level & 80 & 21 & 0 & -5 & 129 \\
\hline \multirow{2}{*}{$\propto E^{-2} e^{-E / 100 \mathrm{TeV}}$} & reco level & 474 & 17707 & $8 \times 10^{6}$ & & \\
\cline { 2 - 8 } & cut level & 77 & 282 & 0 & -4.7 & 56 \\
\hline \multirow{2}{*}{$\propto E^{-2} e^{-E / 30 \mathrm{TeV}}$} & reco level & 315 & 17707 & $8 \times 10^{6}$ & & \\
\cline { 2 - 8 } & cut level & 53 & 658 & 0 & -4.8 & 43 \\
\hline
\end{tabular}

Table 1: Expected numbers of events in one year of data taking for the three samples of simulated events: neutrinos from the Fermi bubbles $\left(\nu_{\text {sig }}\right)$, neutrinos from atmospheric background (conventional plus prompt RQPM) $\left(\nu_{\text {atm }}\right)$ and muons from atmospheric background $\left(\mu_{\text {atm }}\right)$. The cut values $\left(\Lambda\right.$ and $\left.N_{\text {hit }}\right)$ and the numbers of events at the reconstruction level (reco level) and after the cuts (cut level) are also given. The cuts are those optimised for a discovery at $5 \sigma$ C.L., $50 \%$ probability, for a source with a normalisation factor of $K_{0 \nu}=1 \times 10^{-7} \mathrm{GeV} \mathrm{cm}^{-2} \mathrm{~s}^{-1}$.

the number of background events corresponds to an increase of $10 \%$ in the flux limits. In the case of a pure power-law spectrum the contribution of atmospheric muons to the background is negligible.

In Fig. 4 the discovery fluxes at $5 \sigma$ C.L., $50 \%$ probability and $3 \sigma$ C.L., $50 \%$ probability for the neutrino spectra considered (see Eq. (1)) are shown as functions of the observation time. The variation in the discovery fluxes due to the uncertainties in the atmospheric neutrino flux have been evaluated taking into account only the uncertainty on the normalisation factor of the conventional Bartol flux. The RQPM prompt model component contributes with about $46 \%$ and $10 \%$ in the number of neutrino background events after the cuts quoted in Tab. 1 for the pure $E^{-2}$ spectrum and for the exponential 
cutoff spectra, respectively. A $25 \%$ uncertainty in the conventional Bartol atmospheric neutrino flux corresponds to a variation in the discovery flux of about $7 \%$ for $d \Phi_{\nu}^{\infty} / d E$ (see Eq. (1)) and $10 \%$ for $d \Phi_{\nu}^{100} / d E$ and $d \Phi_{\nu}^{30} / d E$. These variations are shown in Fig. 4 as bands around the discovery-flux curves.

If the neutrino normalisation factor is of the order of $1 \times 10^{-7} \mathrm{GeV} \mathrm{cm}^{-2} \mathrm{~s}^{-1}$ the discovery of neutrinos from the Fermi bubbles is expected in about one year of data taking for a $E^{-2}$ neutrino spectrum with a cutoff at $100 \mathrm{TeV}$. The first evidence ( $3 \sigma$ C.L., $50 \%$ probability) could be obtained after a few months of data taking. For the more severe cutoff at $30 \mathrm{TeV}$ the discovery is predicted to be achieved in about 5.5 years and first evidence in about 2.5 years.

Recently an analysis has been performed on 43 months of Fermi data in the energy range $20 \mathrm{GeV}<E_{\gamma}<300 \mathrm{GeV}$ [36]. This analysis covered several regions near the Galactic centre, some of which overlap with the bubble regions. This revealed the presence of an excess in the gamma ray flux around $130 \mathrm{GeV}$. This peak has been interpreted as an indication of dark matter annihilation into two photons. An alternative explanation, put forward in a separate analysis of the same data (in the range $80 \mathrm{GeV}<E_{\gamma}<200 \mathrm{GeV}$ ) [37], is that the excess can be interpreted as a steepening of the power law spectrum of the Fermi bubble gamma rays at around $130 \mathrm{GeV}$. Such a steepening has a significant effect on the results of the present analysis. Therefore, a Fermi bubble neutrino spectrum that follows a power law spectrum according to $d \Phi_{\nu}^{\infty} / d E$ with $K_{0 \nu}(E)=10^{-7} \mathrm{GeV} \mathrm{cm}^{-2} \mathrm{~s}^{-1}$ up to $130 \mathrm{GeV}$ and extends to higher energies proportional to $E^{-2.3}$, has been investigated. In 


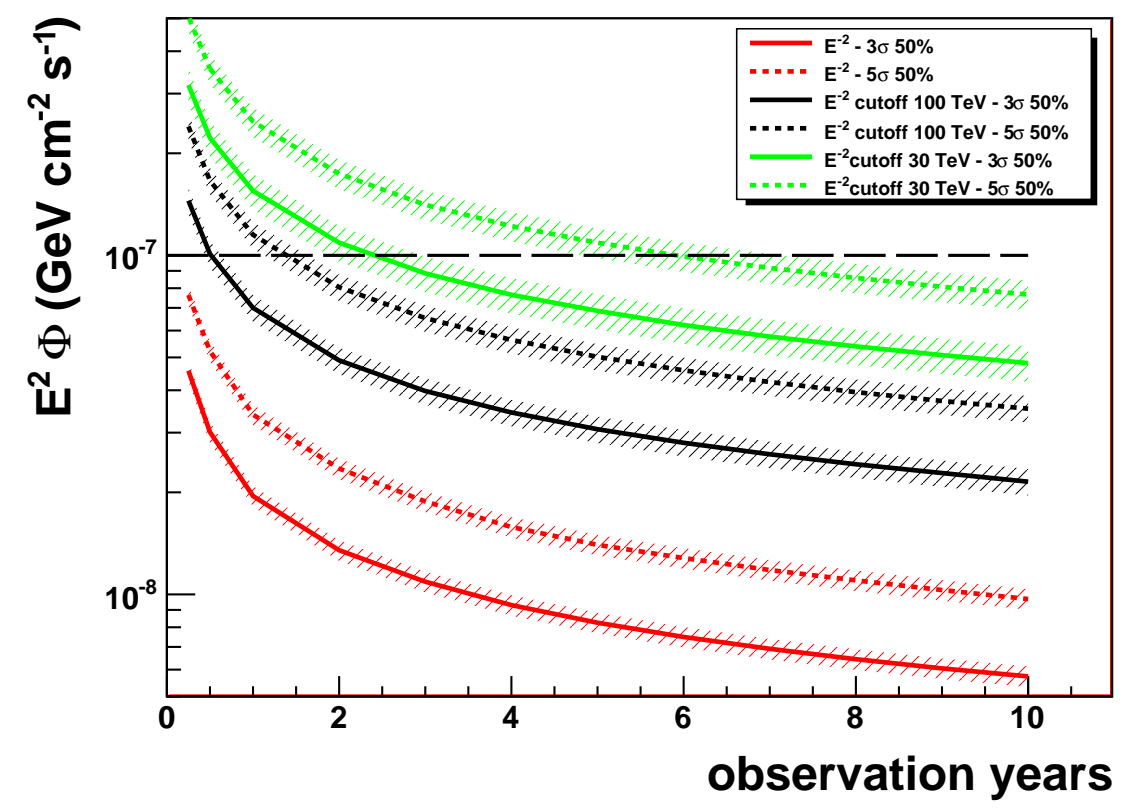

Figure 4: Discovery fluxes as functions of the observation years for $5 \sigma$ C.L., $50 \%$ probability and $3 \sigma$ C.L., $50 \%$ probability, for the three neutrino spectra assumed. The bands represent the variation due to the uncertainty on the normalisation factor of the conventional Bartol neutrino flux. The long-dashed line indicates the predicted neutrino flux estimated in Sec. 4.1.

this case the resulting live time required for discovery is 6 years, which is well in the expected lifetime of the KM3NeT detector.

If no statistically significant excess of neutrino events will be found, upper limits can be set for specific neutrino emission models. In Fig. 5 the average upper flux limits at $90 \%$ of C. L. (computed following the Feldman and Cousins prescription [38]) are reported for the three neutrino spectra investigated. If the observed gamma flux is of purely hadronic origin and the source is fully transparent for gamma rays, the expected neutrino spectrum 


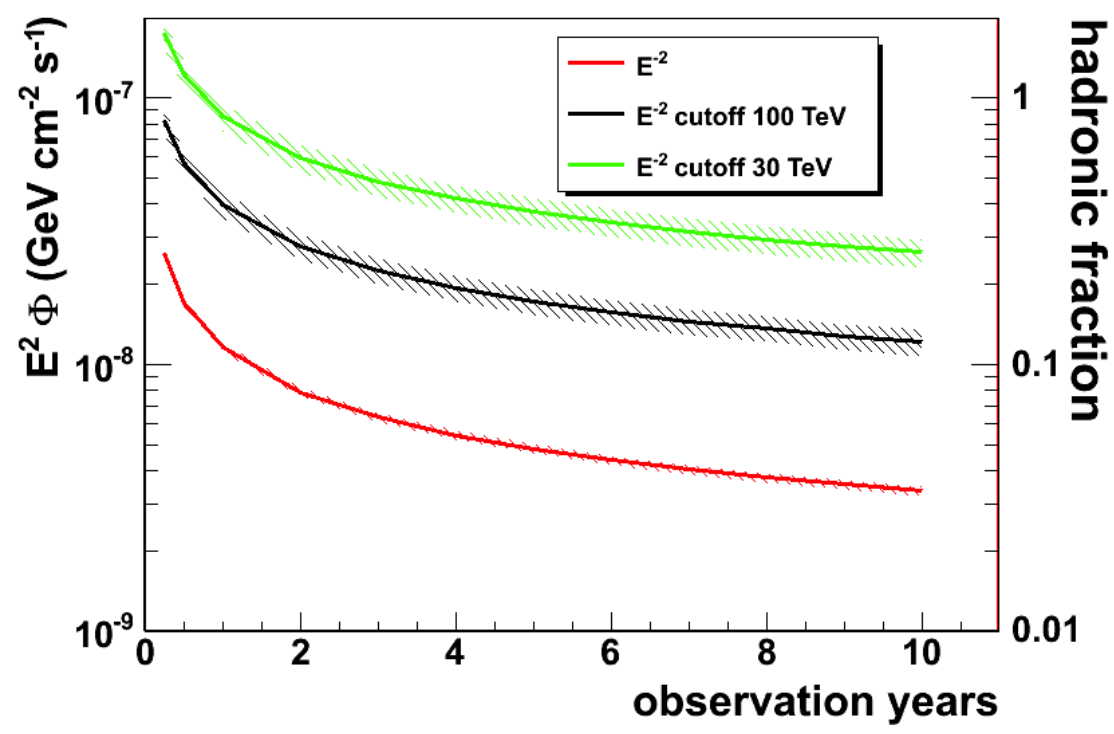

Figure 5: Sensitivity at $90 \%$ C.L. as a function of the observation years for the three neutrino spectra assumed. The right vertical scale indicates the upper limit on the fraction of hadronic emission (see text). The bands represent the variation due to the uncertainty on the normalisation factor of the conventional Bartol neutrino flux.

corresponds to $K_{0 \nu}=1 \times 10^{-7} \mathrm{GeV} \mathrm{cm}^{-2} \mathrm{~s}^{-1}$ (see Sect. 4.1). If the gamma emission is not fully hadronic and the hadronic and non-hadronic emission mechanisms yield the same spectral shapes, upper limits on the percentage of hadronic emission can be extracted from the upper flux limits in Fig. 5 (see right vertical scale).

Since neutrino telescopes have a modular design, the detector concept allows for a staged implementation with continuously increasing science capabilities. Therefore, the capability of the telescope to detect neutrinos from the Fermi bubbles has been explored as a function of an increasing number of 


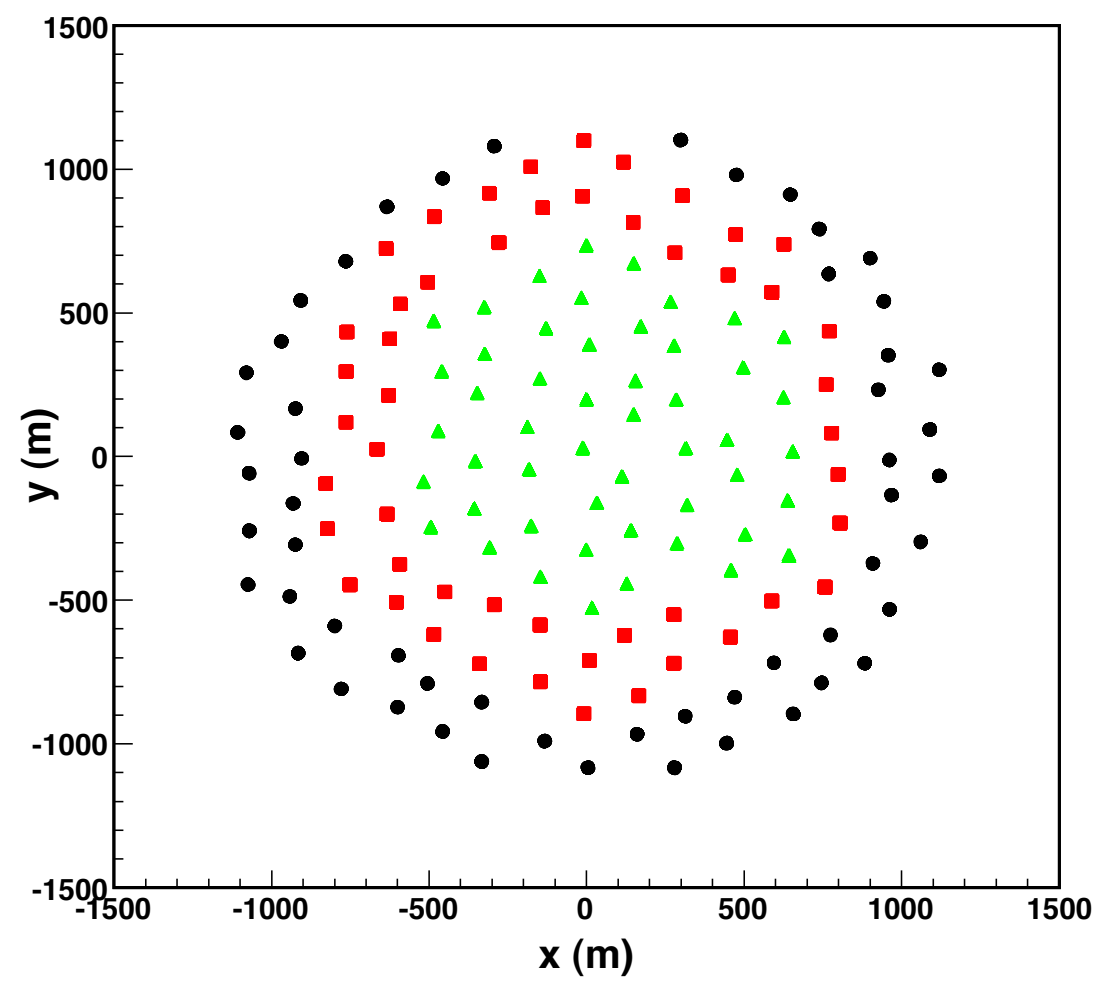

Figure 6: DU positions for a single block of detectors composed of 154 DUs (black circles, red squares and green triangles), 100 DUs (red squares and green triangles) and 50 DUs (green triangles).

DUs. Detector geometries composed of 50, 100 and 154 DUs with the same average DU distance $(180 \mathrm{~m})$ have been investigated (see Fig. 6). These geometries provide increasing active volumes of $1,2.1$ and $3.2 \mathrm{~km}^{3}$ for 50,100 and 154 DUs, respectively. The discovery fluxes have been calculated and are shown in Fig. 7 for $5 \sigma$ C.L., $50 \%$ probability (a) and $3 \sigma$ C.L., $50 \%$ probability (b). The number of years needed for the discovery does not decrease linearly with the increasing number of DUs, being about 8 years for 50 DUs, 

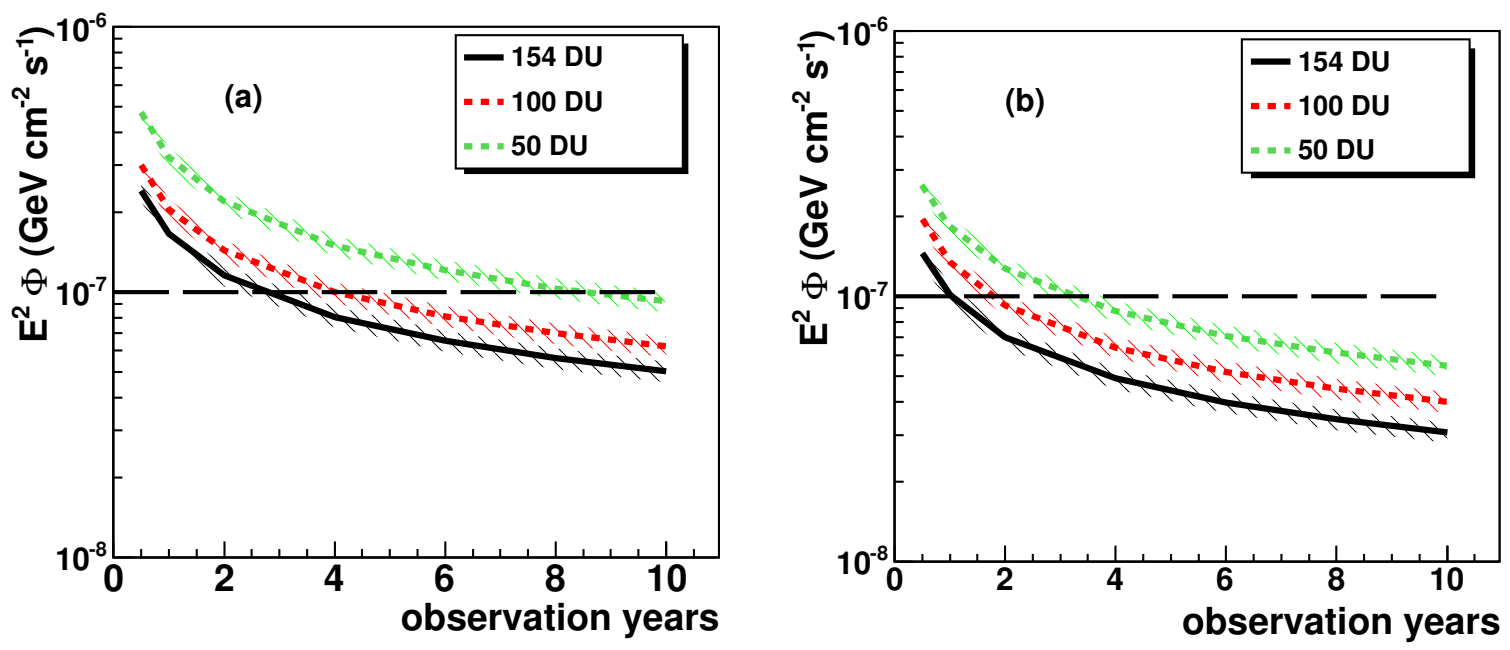

Figure 7: Discovery fluxes as a function of the observation years for a $5 \sigma$ C.L., $50 \%$ probability (a) and $3 \sigma$ C.L., $50 \%$ probability (b) for a $E^{-2}$ neutrino spectrum with a $100 \mathrm{TeV}$ cutoff for different detector sizes (see text). The bands represent the variation due to the uncertainty on the normalisation factor of the conventional Bartol neutrino flux. The long-dashed lines indicate the predicted neutrino flux estimated in Sec. 4.1.

4 years for 100 DUs and 2.5 years for 154 DUs. A neutrino spectrum with normalisation $K_{0 \nu} \approx 1 \times 10^{-7} \mathrm{GeV} \mathrm{cm}^{-2} \mathrm{~s}^{-1}$ with a $100 \mathrm{TeV}$ cutoff energy is assumed here.

\section{Conclusions}

The discovery of an intense gamma-ray flux from two large areas around the Galactic centre, the Fermi bubbles, has stimulated estimates of the capability of neutrino telescopes to discover neutrinos from this region [39, 40]. The Fermi bubbles extend over a large part of the sky and have a significant intensity in gamma rays. Assuming these gamma rays are of hadronic origin and the spectrum extends to the multi-TeV range, this analysis shows that 
high energy neutrinos are expected from near the Galactic centre. Telescopes located in the Mediterranean Sea, such as ANTARES and KM3NeT, have a large visibility of the region around the Galactic centre. Due to its multi$\mathrm{km}^{3}$ scale, the future KM3NeT telescope is the ideal instrument to observe neutrinos from the Fermi bubbles. Note that, due to its location, IceCube has a considerably reduced visibility of the bubbles.

In this paper we present an analysis addressing the sensitivity of KM3NeT to a neutrino flux from the Fermi bubbles that is consistent with the measured gamma ray flux, assuming that the latter fully originates from hadronic processes. Based on a complete chain of Monte Carlo simulations, the time of KM3NeT operation that is required to detect this neutrino flux at a significance of $5 \sigma$ with $50 \%$ probability has been estimated. The result depends strongly on the shape of the neutrino energy spectrum assumed. In the case

of an $E_{\nu}^{-2}$ spectrum with an exponential cutoff at $100 \mathrm{TeV}$, we expect to obtain a $3 \sigma$-evidence in a few months and to claim a discovery after about 1 year of data taking. The non-observation of a signal would severely constrain models of neutrino production through hadronic acceleration processes in the Fermi bubbles.

\section{Acknowledgements}

The research leading to these results has received funding from the European Community's Sixth Framework Programme under contract n ${ }^{\circ} 011937$ and the Seventh Framework Programme under grant agreement ${ }^{\circ} 212525$. 


\section{References}

[1] web site http://fermi.gsfc.nasa.gov/

[2] web site http://www.mpi-hd.mpg.de/hfm/HESS/

[3] web site http://veritas.sao.arizona.edu/

[4] web site http://magic.mppmu.mpg.de/

[5] F. Aharonian et al., Rep. Prog. Phys. 71 (2008) 096901

[6] A.A. Abdo et al., Astrophys. J. 700 (2009) L127

[7] A. Kappes et al., Astrophys. J. 656 (2007) 870; J. Bahcall and E. Waxman, Phys. Rev D64 (2001) 023002

[8] web site http://icecube.wisc.edu/

[9] M. Ageron et al., Nucl. Instr. Meth. A656 (2011) 11

[10] web site http://www.km3net.org/home.php

[11] Meng Su et al., Astrophys. J. 724 (2010) 1044

[12] G. Dobler et al., Astrophys. J. 717 (2010) 825

[13] G. Dobler, Astrophys. J. 750 (2012) 17

[14] P. Mertsch and S. Sarkar, Phys. Rev. Lett. 107 (2011) 091101

[15] Fulai Guo et al., arXiv:1110.0834; Fulai Guo and W.G. Mathews, arXiv:1103.0055 
[16] D. Malyshev, I. Cholis and J.D. Gelfand, Astrophys. J. 722 (2010) 1939

[17] G. Dobler et al., Astrophys. J. 741 (2011) 25

[18] R. Crocker and F. Aharonian, Phys. Rev. Lett. 106 (2011) 101102

[19] F.L. Villante and F. Vissani, Phys. Rev. D 78 (2008) 103007;

F. Vissani and F.L. Villante, Nucl. Inst. Meth. A588 (2008) 123;

F. Vissani, Astropart. Phys. 26 (2006) 310

[20] KM3NeT Technical Design Report, available on http://www.km3net.org/TDR/TDRKM3NeT.pdf (ISBN 978-90-6488-033-9)

[21] P. Kooijman for the KM3NeT collaboration, proceedings of VLVnT11 conference, Erlangen, Germany, Oct. 2011

[22] O. Kavatsyuk, Q. Dorosti-Hasankiadeh and H. Löhner, for the KM3NeT collaboration, proceedings of 6th International Conference on New Developments in Photodetection, Lyon, France, July 2011;

[23] J. Brunner et al., proceedings of the first VLVnT Conference, Amsterdam, Oct. 2003; A. Margiotta, proceedings of VLVnT11 conference, Erlangen, Germany, Oct. 2011.

[24] web site http://geant4.cern.ch/

[25] M. Ageron et al., Astropart. Phys. 31 (2009) 277

[26] A. Heijboer for the ANTARES Collaboration, proceedings of the $31^{s t}$ ICRC, Łódź, Poland, 2009 
[27] IceCube collaboration, proceedings of the $32^{\text {nd }}$ ICRC, Beijng, China, arXiv:1111.2741[astro-ph.HE], 2011

[28] Y. Becherini et al., Astropart. Phys 25 (2006) 1; G. Carminati et al., Comp. Phys. Comm. 179 (2008) 915

[29] C.G.S. Costa, Astropart. Phys. 16 (2001) 193 and references therein

[30] R. Enberg et al., Phys. Rev. D78 (2008) 043005

[31] G.D. Barr et al., Phys. Rev. D70 (2004) 023006

[32] J.A. Aguilar et al., Phys. Lett. B696 (2011) 16

[33] E.V. Bugaev et al., Phys. Rev. D 58 (1998) 054001

[34] J. Ahrens et al., Astropart. Phys. 20 (2004) 507

[35] S. Adrian Martinez, Astrophys. J. Lett. 743 (2011) L14

[36] C. Weniger, arXiv:1204.2797v1, 2012

[37] S. Profumo and T. Linden, arXiv:1204.6047v1[astro-ph.HE], 2012

[38] G.J. Feldman and R.D. Cousins, Phys. Rev. D57 (1998) 3873

[39] C. Lunardini and S. Razzaque, Phys, Rev. Lett. 108 (2012) 221102

[40] I. Cholis, arXiv:1206.1607[astro-ph.HE], 2012 